\title{
SECONDARY CIRCULATION IN GRANULAR FLOW THROUGH NONAXISYMMETRIC HOPPERS*
}

\author{
PIERRE A. GREMAUD ${ }^{\dagger}$, JOHN V. MATTHEWS ${ }^{\ddagger}$, AND DAVID G. SCHAEFFER ${ }^{\S}$
}

\begin{abstract}
Jenike's radial solution, widely used in the design of materials-handling equipment, is a similarity solution of steady-state continuum equations for the flow under gravity of granular material through an infinite, right-circular cone. In this paper we study how the geometry of the hopper influences this solution. Using perturbation theory, we compute a first-order correction to the (steady-state) velocity resulting from a small change in hopper geometry, either distortion of the cross section or tilting away from vertical. Unlike for the Jenike solution, all three components of the correction velocity are nonzero; i.e., there is secondary circulation in the perturbed flow.
\end{abstract}

Key words. granular, similarity solution, perturbation theory

AMS subject classifications. 65L80, 35J65, 76T25

DOI. $10.1137 /$ S0036139903415124

1. Introduction. In manufacturing industries, raw materials are stored in granular form in a silo, and when needed, they are expected to flow out of the silo under gravity through a hopper. Problems in the discharge process are frequent and expensive; see, e.g., [8]. As demonstrated by a Rand Corporation study [9], these problems are symptomatic of our poor understanding of the behavior of granular materials. ${ }^{1}$

Jenike's radial solution is a central component of silo design. Despite its importance, this solution is subject to many severe restrictions:

1. Granular material is modeled as a continuum, with an ad hoc constitutive law.

2. The flow is assumed to be steady.

3. The flow domain, a mathematical idealization, is an infinite cone, given in spherical polar coordinates by the formula

$$
\left\{(r, \theta, \phi): 0<r<\infty, 0 \leq \theta<\theta_{w}\right\} \quad\left(\theta_{w}=\text { constant }\right) .
$$

4. Only similarity solutions are considered.

${ }^{*}$ Received by the editors July 7, 2003; accepted for publication (in revised form) July 22, 2003; published electronically December 31, 2003.

http://www.siam.org/journals/siap/64-2/41512.html

$\dagger$ Department of Mathematics and Center for Research in Scientific Computation, North Carolina State University, Raleigh, NC 27695-8205 (gremaud@math.ncsu.edu). The research of this author was partially supported by the Army Research Office through grant DAAD19-99-1-0188 and by the National Science Foundation through grant DMS-9818900.

${ }_{\ddagger}^{\ddagger}$ Department of Mathematics, Duke University, Durham, NC 27708-0320 (jvmatthe@math.duke. edu). The research of this author was partially supported by the National Science Foundation through grant DMS-9983320.

$\S$ Department of Mathematics and Center for Nonlinear and Complex Systems, Duke University, Durham, NC 27708-0320 (dgs@math.duke.edu). The research of this author was partially supported by the National Science Foundation through grant DMS-9803305.

${ }^{1}$ The study compared the design output and the actual output of a total of 60 manufacturing plants in various industries, 22 that were based primarily on liquids-processing technology and 38 on solids-processing technology. On average, the liquids-processing plants produced at $84 \%$ of design capacity while the solids-processing plants produced at only $63 \%$ of design capacity. To quote Merrow [9], "In economic terms, the difference between $63 \%$ of design and $84 \%$ is very large. It implies a capital cost per unit of output about one-third higher for the solids-processing plants, on the basis of poor performance alone. In addition, poor performance is inevitably associated with higher operating and maintenance costs per unit of product." Moreover, the standard deviation of the solids-processing data set was much greater, indicative of our difficulties in predicting the behavior of granular solids. 
In this paper we relax restrictions 3 and 4 partly. Specifically, we generalize the domain to an infinite pyramidal hopper described by the inequality

$$
0 \leq \theta<\theta_{w}+\epsilon \cos m \phi
$$

where $\epsilon$ is a small parameter and $m$ is a positive integer. Assuming a perturbation series

$$
v^{(0)}+\epsilon v^{(1)}+\cdots
$$

for the flow velocity in the domain (1.1), where $v^{(0)}$ is Jenike's solution, we derive a linear PDE for the first-order correction $v^{(1)}$. The $r$-dependence of $v^{(1)}$ still has similarity form, and the $\phi$-dependence may be handled by separation of variables. In this way we reduce solving the PDE for $v^{(1)}$ to solving a two-point boundary problem on the interval $0<\theta<\theta_{w}$.

In Jenike's solution, only the radial component $v_{r}^{(0)}$ of the velocity is nonzero. By contrast, all three components of the correction velocity $v^{(1)}$ are nonzero. In other words, distortion of the conical domain leads to secondary circulation. For example, in Figure 5.1 below, the flow in the $\theta, \phi$-directions is shown for a circular hopper that is tilted slightly to the right, and in Figure 5.2, for a slightly distorted vertical hopper.

Circulation was previously observed by Williams and Rege in discrete element simulations of granular systems [11], [13]. While a connection between such timedependent, discrete simulations and the steady-state continuum theory below is unclear, the similarities are uncanny. Both find a secondary circulation in essentially two-dimensional granular systems undergoing a uniform compression.

The outline of the paper is as follows. In section 2, the governing equations are recalled together with Jenike's construction of similarity solutions in conical domains. For nonaxisymmetric domains of the type (1.1), the problem is then linearized about Jenike's solution in section 3. The resulting system is discretized in section 4 . Numerical results and discussion are offered in section 5 .

\section{The model.}

2.1. Governing equations and boundary conditions. The unknowns are the 3 -component velocity vector $v$, the $3 \times 3$ symmetric stress tensor $T$, and a scalar plasticity coefficient $\lambda$. (The density $\rho$ is a constant.) In total, there are $3+6+1=$ 10 unknown functions. In writing the equations for these variables, we need the strain rate tensor $V=-1 / 2\left(\nabla v+\nabla v^{T}\right)$ and the deviatoric part of the stress tensor $\operatorname{dev} T=T-\frac{1}{3}(\operatorname{tr} T)$ I. Note the sign convention: $V$ measures the compression rate of the material; analogously, positive eigenvalues of $T$ correspond to compressive stresses. This sign convention reflects the fact that granular materials disintegrate under tensile stresses.

Following [12], we require that these variables satisfy

$$
\begin{aligned}
\nabla \cdot T & =\rho g, \\
V & =\lambda \operatorname{dev} T, \\
|\operatorname{dev} T|^{2} & =2 s^{2}(\operatorname{tr} T / 3)^{2},
\end{aligned}
$$

where $g$ is the (vector) acceleration of gravity, $|\cdot|$ denotes the Frobenius norm

$$
|T|^{2}=\sum_{i, j=1}^{3} T_{i j}^{2}=\operatorname{tr} T^{2}
$$


(the latter equality only for symmetric tensors), and $s=\sin \delta$, with $\delta$ being the angle of internal friction of the material under consideration (see [10]). Equation (2.1) expresses force balance; i.e., Newton's second law with inertia neglected because the flow is assumed slow; it is equivalent to three scalar equations. Equations (2.2) and (2.3) are constitutive laws, the alignment condition and the von Mises yield condition, respectively; they are equivalent to six and to one scalar equations, respectively. Thus (2.1)-(2.3) is a determined system, ten equations for ten unknowns. Since (2.3) contains no derivatives, this system has a differential-algebraic character. Taking the trace of (2.2), we see that $\operatorname{div} v=-\operatorname{tr} V=0$; thus, incompressibility is part of the constitutive assumptions. Incidentally, for a solution to be physical, the function $\lambda$ in (2.2) must satisfy $\lambda \geq 0$ everywhere; otherwise, friction would be adding energy to the system rather than dissipating it. In fact, we want $\lambda$ to be strictly positive since one of the assumptions underlying the derivation of (2.1)-(2.3) is that material is actually deforming.

We seek solutions of (2.1)-(2.3) in a pyramidal domain, expressed in spherical polar coordinates as

$$
\Omega=\{(r, \theta, \phi): 0 \leq \theta<\mathcal{C}(\phi)\},
$$

where $\mathcal{C}$ is a given smooth $2 \pi$-periodic function. Such a domain represents a mathematical idealization of a converging hopper, in general, a nonaxisymmetric one.

On the boundary $\partial \Omega=\{(r, \mathcal{C}(\phi), \phi)\}$, wall impenetrability imposes one boundary condition on the velocity; i.e.,

$$
v_{N}=0,
$$

where $v_{N}$ is the normal velocity. Two additional boundary conditions come from Coulomb's law of sliding friction. The surface traction $\tau$-i.e., the force exerted by the wall on the material - is given by

$$
\tau_{i}=\sum_{j=1}^{3} T_{i j} N_{j},
$$

where $N$ is the unit interior normal to $\partial \Omega$. If the vector $\tau$ has normal component $\tau_{N}$ and tangential component $\tau_{T}=\tau-\tau_{N} N$, then we require that

$$
\tau_{T}=-\mu_{w} \tau_{N}(v /|v|),
$$

where $\mu_{w}$ is the coefficient of friction between the wall and the material. Note the following: (i) If $T$ is positive definite (i.e., if all stresses are compressive), then $\tau_{N}>0$. (ii) While $\tau_{N}$ is a scalar, $\tau_{T}$ is effectively a two-component vector; thus, (2.6) is equivalent to two scalar equations. (iii) Because of (2.5), the velocity $v$ is tangential to $\partial \Omega$; we are assuming that $v \neq 0$ at the boundary.

2.2. Jenike's similarity solution. Suppose that the domain (2.4) is axisymmetric; i.e., suppose

$$
\Omega=\left\{(r, \theta, \phi): 0 \leq \theta<\theta_{w}\right\},
$$

where $\theta_{w}$ is a constant. In this case Jenike [7] found that (2.1)-(2.3) have solutions that are independent of $\phi$ and have a similarity dependence on $r$,

$$
v^{(0)}(r, \theta)=r^{-2} \hat{v}^{(0)}(\theta), \quad T^{(0)}(r, \theta)=r \hat{T}^{(0)}(\theta) .
$$


(Here and below, a hat above a variable indicates a function that depends on $\theta$ alone.) Moreover, only the radial component of velocity is nonzero; i.e., $v_{\theta}^{(0)}=v_{\phi}^{(0)}=0$. Similarly, $T_{r \phi}^{(0)}=T_{\theta \phi}^{(0)}=0$. Indeed, all components of $T$ can be expressed in terms of two scalar variables, the so-called Sokolovskii variables [10]: the mean stress $p^{(0)}=$ $\operatorname{tr} T^{(0)} / 3$ and an angle $\psi$; specifically,

$$
\operatorname{dev} T^{(0)}=s p^{(0)}\left[\begin{array}{ccc}
-\frac{2}{\sqrt{3}} \cos 2 \psi & -\sin 2 \psi & 0 \\
-\sin 2 \psi & \frac{1}{\sqrt{3}} \cos 2 \psi & 0 \\
0 & 0 & \frac{1}{\sqrt{3}} \cos 2 \psi
\end{array}\right],
$$

where $p^{(0)}=r \hat{p}^{(0)}$ and the function $\psi$, like $\hat{p}^{(0)}$, depends only on $\theta$.

The boundary conditions (2.5), (2.6) may be written more explicitly when $\Omega$ is axisymmetric. Equation (2.5) reduces to

$$
v_{\theta}=0
$$

Let us decompose the vector equation (2.6) into a direction and a magnitude. Regarding the direction, the vectors $\tau_{T}$ and $v$ are parallel if

$$
T_{r \theta} v_{\phi}-T_{\theta \phi} v_{r}=0 .
$$

Jenike's solution satisfies both (2.10) and (2.11) trivially. The two sides of (2.6) have equal magnitude if

$$
T_{r \theta}+\mu_{w} T_{\theta \theta}=0 .
$$

We briefly summarize the construction of Jenike's solution, referring to [12] for more details. The ansatz (2.9) arranges that (2.3) holds automatically. On substitution into (2.1), we obtain a first-order $2 \times 2$ system of ODEs for $\hat{p}^{(0)}$ and $\psi$. This system has a regular singular point at $\theta=0$, and one boundary condition comes from requiring that the solution be regular there; the other boundary condition comes from (2.12). Thus the stresses are determined as the solution of a two-point boundary-value problem. (In axial symmetry, the stress equations decouple from the velocity.) Once the stresses are known, (2.2) reduces to a linear first-order ODE for $\hat{v}_{r}^{(0)}$. The velocity is determined only up to a multiplicative constant, but the normalization of the velocity will scale out of the calculations below.

Incidentally, for Jenike's solution the plasticity coefficient $\lambda$ in (2.2), which cancels out in the derivation of the equation for $\hat{v}_{r}^{(0)}$, has the form

$$
\lambda^{(0)}(r, \theta)=r^{-4} \hat{\lambda}^{(0)}(\theta) .
$$

Using (2.2), the function $\hat{\lambda}^{(0)}$ may be determined from $\hat{v}_{r}^{(0)}$.

\section{Linearized analysis for a nearly axisymmetric domain.}

3.1. Derivation of linearized differential equations. Equations (2.1)-(2.3), a $10 \times 10$ nonlinear DAE system that is elliptic in the sense of Agmon, Douglis, and Nirenberg [1], present formidable mathematical and numerical challenges. In this paper, we consider a simplified problem that prepares the way for computations with the full problem on a general domain. 
Suppose the function $\mathcal{C}$ specifying the boundary of $\Omega$ in (2.4) has the expansion

$$
\mathcal{C}(\phi)=\theta_{w}+\epsilon \cos (m \phi)+\mathcal{O}\left(\epsilon^{2}\right),
$$

where $m$ is a positive integer. For example, a slightly tilted (circular) cone admits such a representation with $m=1$, where $\epsilon$ measures the angle of tilt; likewise for a (vertical) pyramidal hopper having a slightly elliptical cross section, with $m=2$.

An expansion of the solution

$$
v=v^{(0)}+\epsilon v^{(1)}+\mathcal{O}\left(\epsilon^{2}\right), \quad T=T^{(0)}+\epsilon T^{(1)}+\mathcal{O}\left(\epsilon^{2}\right)
$$

is sought, where $v^{(0)}, T^{(0)}$ are equal to Jenike's radial solution; see (2.8). Substituting (3.2) into (2.1)-(2.3), we derive the equations for the first-order perturbation

$$
\begin{aligned}
\nabla \cdot T^{(1)} & =0, \\
V^{(1)} & =\lambda^{(1)} \operatorname{dev} T^{(0)}+\lambda^{(0)} \operatorname{dev} T^{(1)}, \\
\operatorname{tr}\left(\operatorname{dev} T^{(0)} \operatorname{dev} T^{(1)}\right) & =2 s^{2} p^{(0)} p^{(1)},
\end{aligned}
$$

where $p^{(i)}=\operatorname{tr} T^{(i)} / 3, i=0,1$, are the mean stresses.

The correction velocity $v^{(1)}$ has the same $r$-dependence as the Jenike solution (although all three components of $v^{(1)}$ may be nonzero), and its $\phi$-dependence can be obtained through separation of variables. Indeed, suppose each component of $v^{(1)}$ has the form

$$
v_{j}^{(1)}=r^{-2} \hat{v}_{j}^{(1)}(\theta) \operatorname{trig} m \phi,
$$

where trig $m \phi$ denotes either $\cos m \phi$ or $\sin m \phi$. In order to satisfy the appropriately modified version of the boundary condition $(2.10)$ on the perturbed domain, $v_{\theta}^{(1)}$ will have to be in phase with (3.1); i.e., we need

$$
v_{\theta}^{(1)}=r^{-2} \hat{v}_{\theta}^{(1)}(\theta) \cos m \phi .
$$

It is readily seen that if

$$
v_{r}^{(1)}=r^{-2} \hat{v}_{r}^{(1)}(\theta) \cos m \phi \quad \text { and } \quad v_{\phi}^{(1)}=r^{-2} \hat{v}_{\phi}^{(1)}(\theta) \sin m \phi,
$$

then all terms in

$$
\nabla \cdot v^{(1)}=\partial_{r} v_{r}^{(1)}+2 r^{-1} v_{r}^{(1)}+r^{-1} \partial_{\theta} v_{\theta}^{(1)}+r^{-1} \cot \theta v_{\theta}^{(1)}+r^{-1} \csc \theta \partial_{\phi} v_{\phi}^{(1)}
$$

are proportional to $r^{-3} \cos m \phi$; i.e., variables separate in the equation $\nabla \cdot v=0$.

Tables 3.1-3.3 help systematize the elimination of $\phi$-dependence in (3.3)-(3.5) with separation of variables. The appropriate $r$ - and $\phi$-dependencies for the scalar $p^{(1)}$, for the vector $v^{(1)}$, and for the tensor $T^{(1)}$ are indicated in Table 3.1. (Note that symmetric $3 \times 3$ tensors are represented as vectors in $\mathbf{R}^{6}$, the components being enumerated in the order shown.) In Table 3.2 we record, for the reader's convenience, the expressions in spherical coordinates for four differential operators that occur in these equations.

The main point, which makes separation of variables work in this problem, is that the $\theta$-dependent part of each of these linear operators is given by

$$
\begin{aligned}
\widehat{\nabla p} & =\left(g_{1} \partial_{\theta}+g_{0}\right) \hat{p}, \\
\widehat{\nabla \cdot v} & =\left(d_{1}^{T} \partial_{\theta}+d_{0}^{T}\right) \hat{v}, \\
\hat{V} & =-\left(G_{1} \partial_{\theta}+G_{0}\right) \hat{v}, \\
\widehat{\nabla \cdot T} & =\left(D_{1} \partial_{\theta}+D_{0}\right) \hat{T},
\end{aligned}
$$


TABLE 3.1

The $r$ - and $\phi$-dependence of scalars, vectors, and tensors in separation of variables.

\begin{tabular}{|c|c|c|c|c|c|}
\hline Scalars : & $p=$ & $\hat{p}(\theta) \cos m \phi$ & & & $\begin{array}{l}\hat{T}_{r r}(\theta) \cos m \phi \\
\hat{T}_{r \theta}(\theta) \cos m \phi\end{array}$ \\
\hline Vectors: & $v=\frac{1}{r^{2}}$ & $\begin{array}{l}\hat{v}_{r}(\theta) \cos m \phi \\
\hat{v}_{\theta}(\theta) \cos m \phi \\
\hat{v}_{\phi}(\theta) \sin m \phi\end{array}$ & Tensors & $T=r$ & $\begin{array}{c}\hat{T}_{\theta \theta}(\theta) \cos m \phi \\
\hat{T}_{r \phi}(\theta) \sin m \phi \\
\hat{T}_{\theta \phi}(\theta) \sin m \phi \\
\hat{T}_{\phi \phi}(\theta) \cos m \phi\end{array}$ \\
\hline
\end{tabular}

TABLE 3.2

Differential operators in spherical coordinates.

$$
\begin{aligned}
& \nabla p=\left[\partial_{r} p, r^{-1} \partial_{\theta} p, r^{-1} \csc \theta \partial_{\phi} p\right]^{T} \\
& \nabla \cdot v=\partial_{r} v_{r}+2 r^{-1} v_{r}+r^{-1} \partial_{\theta} v_{\theta}+r^{-1} \cot \theta v_{\theta}+r^{-1} \csc \theta \partial_{\phi} v_{\phi} \\
& V=\left[\begin{array}{c}
V_{r r} \\
V_{r \theta} \\
V_{\theta \theta} \\
V_{r \phi} \\
V_{\theta \phi} \\
V_{\phi \phi}
\end{array}\right]=-\left[\begin{array}{c}
\partial_{r} v_{r} \\
\frac{1}{2}\left(r^{-1} \partial_{\theta} v_{r}-r^{-1} v_{\theta}+\partial_{r} v_{\theta}\right) \\
r^{-1}\left(v_{r}+\partial_{\theta} v_{\theta}\right) \\
\frac{1}{2}\left(r^{-1} \csc \theta \partial_{\phi} v_{r}-r^{-1} v_{\phi}+\partial_{r} v_{\phi}\right) \\
\frac{1}{2} r^{-1}\left(\partial_{\theta} v_{\phi}-\cot \theta v_{\phi}+\csc \theta \partial_{\phi} v_{\theta}\right) \\
r^{-1}\left(v_{r}+\cot \theta v_{\theta}+\csc \theta \partial_{\phi} v_{\phi}\right)
\end{array}\right] \\
& \nabla \cdot T=\left[\begin{array}{c}
\partial_{r} T_{r r}+r^{-1} \csc \theta \partial_{\phi} T_{r \phi}+r^{-1} \partial_{\theta} T_{r \theta}+r^{-1}\left(2 T_{r r}-T_{\phi \phi}-T_{\theta \theta}+T_{r \theta} \cot \theta\right) \\
\partial_{r} T_{r \theta}+r^{-1} \csc \theta \partial_{\phi} T_{\theta \phi}+r^{-1} \partial_{\theta} T_{\theta \theta}+r^{-1}\left(3 T_{r \theta}+\left(T_{\theta \theta}-T_{\phi \phi}\right) \cot \theta\right) \\
\partial_{r} T_{r \phi}+r^{-1} \csc \theta \partial_{\phi} T_{\phi \phi}+r^{-1} \partial_{\theta} T_{\theta \phi}+r^{-1}\left(3 T_{r \phi}+2 T_{\theta \phi} \cot \theta\right)
\end{array}\right]
\end{aligned}
$$

where $g_{1}, g_{0}, \ldots, D_{0}$ are the matrices given in Table 3.3 .

The calculation needed to verify (3.8b) was described above; the other equations may be verified similarly. Incidentally, (3.8a) may be derived by substituting $T=p I$ in $(3.8 \mathrm{~d})$, and $(3.8 \mathrm{~b})$ may be derived by taking the trace of $(3.8 \mathrm{c})$.

With this notation, (3.3)-(3.5) reduces to a system of ODEs in $\theta$,

$$
\begin{aligned}
\left(D_{1} \partial_{\theta}+D_{0}\right) \hat{T}^{(1)} & =0, \\
-\left(G_{1} \partial_{\theta}+G_{0}\right) \hat{v}^{(1)} & =\hat{\lambda}^{(1)} \operatorname{dev} \hat{T}^{(0)}+\hat{\lambda}^{(0)} \operatorname{dev} \hat{T}^{(1)}, \\
\operatorname{tr}\left(\operatorname{dev} \hat{T}^{(0)} \operatorname{dev} \hat{T}^{(1)}\right) & =2 s^{2} \hat{p}^{(0)} \hat{p}^{(1)} .
\end{aligned}
$$

Recalling the representation of symmetric tensors as 6-component vectors, we observe that the left-hand side of (3.11) may be rewritten as an inner product

$$
\operatorname{tr}\left(\operatorname{dev} \hat{T}^{(0)} \operatorname{dev} \hat{T}^{(1)}\right)=\operatorname{dev} \hat{T}^{(0) T} \mathcal{M} \operatorname{dev} \hat{T}^{(1)},
$$

where $\mathcal{M}$ is the $6 \times 6$ matrix

$$
\mathcal{M}=\operatorname{diag}(1,2,1,2,2,1)
$$

thus we may rewrite (3.11) as

$$
\operatorname{dev} \hat{T}^{(0) T} \mathcal{M} \operatorname{dev} \hat{T}^{(1)}=2 s^{2} \hat{p}^{(0)} \hat{p}^{(1)} .
$$


TABle 3.3

Matrices in (3.8).

$$
\begin{aligned}
& g_{1}=\left[\begin{array}{lll}
0 & 1 & 0
\end{array}\right]^{T} \quad g_{0}=\left[\begin{array}{lll}
1 & 0 & \frac{m}{\sin \theta}
\end{array}\right]^{T} \\
& d_{1}=\left[\begin{array}{lll}
0 & 1 & 0
\end{array}\right]^{T} \quad d_{0}=\left[\begin{array}{lll}
0 & \cot \theta & \frac{m}{\sin \theta}
\end{array}\right]^{T} \\
& G_{1}=\left[\begin{array}{ccc}
0 & 0 & 0 \\
1 / 2 & 0 & 0 \\
0 & 1 & 0 \\
0 & 0 & 0 \\
0 & 0 & 1 / 2 \\
0 & 0 & 0
\end{array}\right] \quad G_{0}=\left[\begin{array}{ccc}
-2 & 0 & 0 \\
0 & -3 / 2 & 0 \\
1 & 0 & 0 \\
-\frac{m}{2 \sin \theta} & 0 & -3 / 2 \\
0 & -\frac{m}{2 \sin \theta} & -\frac{\cot \theta}{2} \\
1 & \cot \theta & \frac{m}{\sin \theta}
\end{array}\right] \\
& D_{1}=\left[\begin{array}{llllll}
0 & 1 & 0 & 0 & 0 & 0 \\
0 & 0 & 1 & 0 & 0 & 0 \\
0 & 0 & 0 & 0 & 1 & 0
\end{array}\right] \quad D_{0}=\left[\begin{array}{ccccccc}
3 & \cot \theta & -1 & \frac{m}{\sin \theta} & 0 & -1 \\
0 & 4 & \cot \theta & 0 & \frac{m}{\sin \theta} & -\cot \theta \\
0 & 0 & 0 & 4 & 2 \cot \theta & -\frac{m}{\sin \theta}
\end{array}\right]
\end{aligned}
$$

Let us show that the deviatoric stresses in (3.9), (3.10), (3.12) can be eliminated from these equations to obtain

$$
\begin{array}{r}
\left(D_{1} \partial_{\theta}+D_{0}\right)\left(-\frac{1}{\hat{\lambda}^{(0)}}\left(G_{1} \partial_{\theta}+G_{0}\right) \hat{v}^{(1)}-\frac{\hat{\lambda}^{(1)}}{\left(\hat{\lambda}^{(0)}\right)^{2}} \hat{V}^{(0)}\right)+\left(g_{1} \partial_{\theta}+g_{0}\right) \hat{p}^{(1)}=0 \\
\left(d_{1}^{T} \partial_{\theta}+d_{0}^{T}\right) \hat{v}^{(1)}=0
\end{array}
$$

where in $(3.13)$

$$
\hat{\lambda}^{(1)}=-\frac{1}{2 s^{2}} \frac{1}{\left(\hat{p}^{(0)}\right)^{2} \hat{\lambda}^{(0)}} \hat{V}^{(0) T} \mathcal{M}\left(G_{1} \partial_{\theta}+G_{0}\right) \hat{v}^{(1)}-\frac{\hat{\lambda}^{(0)}}{\hat{p}^{(0)}} \hat{p}^{(1)} .
$$

Equation (3.14) follows on taking the trace of (3.10). Next, we rewrite (3.10) as

$$
\operatorname{dev} \hat{T}^{(1)}=-\frac{1}{\hat{\lambda}^{(0)}}\left(G_{1} \partial_{\theta}+G_{0}\right) \hat{v}^{(1)}-\frac{\hat{\lambda}^{(1)}}{\left(\hat{\lambda}^{(0)}\right)^{2}} \hat{V}^{(0)}
$$

where we have eliminated $\operatorname{dev} \hat{T}^{(0)}$ using the relation $\hat{V}^{(0)}=\hat{\lambda}^{(0)} \operatorname{dev} \hat{T}^{(0)}$ - effectively, (2.2) for Jenike's solution. Recalling that $\hat{T}^{(1)}=\operatorname{dev} \hat{T}^{(1)}+\hat{p}^{(1)} I$, we substitute (3.16) into (3.9) to derive (3.13). Similarly, (3.15) follows on substituting (3.16) into (3.12) and rearranging.

As a final simplification, we substitute (3.15) into (3.13), obtaining the linear, homogeneous system of ODEs

$$
\begin{aligned}
-\left(A_{2} \partial_{\theta \theta}+A_{1} \partial_{\theta}+A_{0}\right) \hat{v}^{(1)}+\left(b_{1} \partial_{\theta}+b_{0}\right) \hat{p}^{(1)} & =0 \\
\left(d_{1}^{T} \partial_{\theta}+d_{0}^{T}\right) \hat{v}^{(1)} & =0
\end{aligned}
$$

where, with the definition

$$
P=I-\frac{1}{2 s^{2}\left(\hat{p}^{(0)}\right)^{2}\left(\hat{\lambda}^{(0)}\right)^{2}} \hat{V}^{(0)} \hat{V}^{(0) T} \mathcal{M}
$$


the coefficient matrices are given by

$$
\begin{aligned}
A_{2} & =\frac{1}{\hat{\lambda}^{(0)}} D_{1} P G_{1}, \\
A_{1} & =\frac{1}{\hat{\lambda}^{(0)}}\left(D_{0} P G_{1}+D_{1} P G_{0}\right)+D_{1} \partial_{\theta}\left(\frac{1}{\hat{\lambda}^{(0)}} P G_{1}\right), \\
A_{0} & =\frac{1}{\hat{\lambda}^{(0)}} D_{0} P G_{0}+D_{1} \partial_{\theta}\left(\frac{1}{\hat{\lambda}^{(0)}} P G_{0}\right), \\
b_{1} & =g_{1}+D_{1} \frac{\hat{V}^{(0)}}{\hat{p}^{(0)} \hat{\lambda}^{(0)}}, \\
b_{0} & =g_{0}+\left(D_{1} \partial_{\theta}+D_{0}\right) \frac{\hat{V}^{(0)}}{\hat{p}^{(0)} \hat{\lambda}^{(0)}} .
\end{aligned}
$$

These matrices depend on $\theta$ and in fact several are singular as $\theta \rightarrow 0$. In Corollary 4.2 below, we show that this system has a six-dimensional solution space.

The combination $\hat{V}^{(0)} /\left(\hat{p}^{(0)} \hat{\lambda}^{(0)}\right)$, which occurs in various places in the above formulas, admits a convenient representation; i.e., combining (2.2) and (2.9), we deduce that

$$
\frac{1}{\hat{p}^{(0)} \hat{\lambda}^{(0)}} \hat{V}^{(0)}=s\left[\begin{array}{c}
-\frac{2}{\sqrt{3}} \cos 2 \psi \\
-\sin 2 \psi \\
\frac{1}{\sqrt{3}} \cos 2 \psi \\
0 \\
0 \\
\frac{1}{\sqrt{3}} \cos 2 \psi
\end{array}\right] .
$$

The following supplementary information will be needed in section 4 .

LEMмA 3.1. Under the reflection $\theta \mapsto-\theta$, the functions in separation of variables have the parities

$$
\begin{aligned}
& \hat{p}(-\theta)=(-1)^{m} \hat{p}(\theta), \\
& \hat{v}_{r}^{(1)}(-\theta)=(-1)^{m} \hat{v}_{r}^{(1)}(\theta), \\
& \hat{v}_{\theta}^{(1)}(-\theta)=(-1)^{m+1} \hat{v}_{\theta}^{(1)}(\theta), \\
& \hat{v}_{\phi}^{(1)}(-\theta)=(-1)^{m+1} \hat{v}_{\phi}^{(1)}(\theta) .
\end{aligned}
$$

Proof. The reflection $\theta \mapsto-\theta$ and the rotation $\phi \mapsto \phi+\pi$ are different representations of the same mapping. Therefore, since $p$ is a scalar,

$$
\hat{p}(-\theta) \cos m \phi=\hat{p}(\theta) \cos m(\phi+\pi)=(-1)^{m} \hat{p}(\theta) \cos m \phi,
$$

which proves (3.20a). Equation (3.20b) follows from the same argument since $v_{r}$ transforms as a scalar under changes in the angular coordinates. Rather than analyze the parities of $v_{\theta}$ and $v_{\phi}$, we prefer an indirect argument. Since $\nabla \cdot v^{(1)}$ is a scalar, $\nabla \cdot v^{(1)}$ has parity $(-1)^{m}$ under the reflection $\theta \mapsto-\theta$, and on inspecting (3.7), we deduce $(3.20 \mathrm{c}),(3.20 \mathrm{~d})$.

Incidentally, although we shall not need that information below, we remark that under this reflection $\hat{T}_{r r}, \hat{T}_{\theta \theta}, \hat{T}_{\theta \phi}$, and $\hat{T}_{\phi \phi}$ have parity $(-1)^{m}$, while $\hat{T}_{r \theta}$ and $\hat{T}_{\phi r}$ have parity $(-1)^{m+1}$. 
TABLE 3.4

Leading orders in the expansions at $\theta=0$ of the coefficient matrices of (3.17)-(3.18).

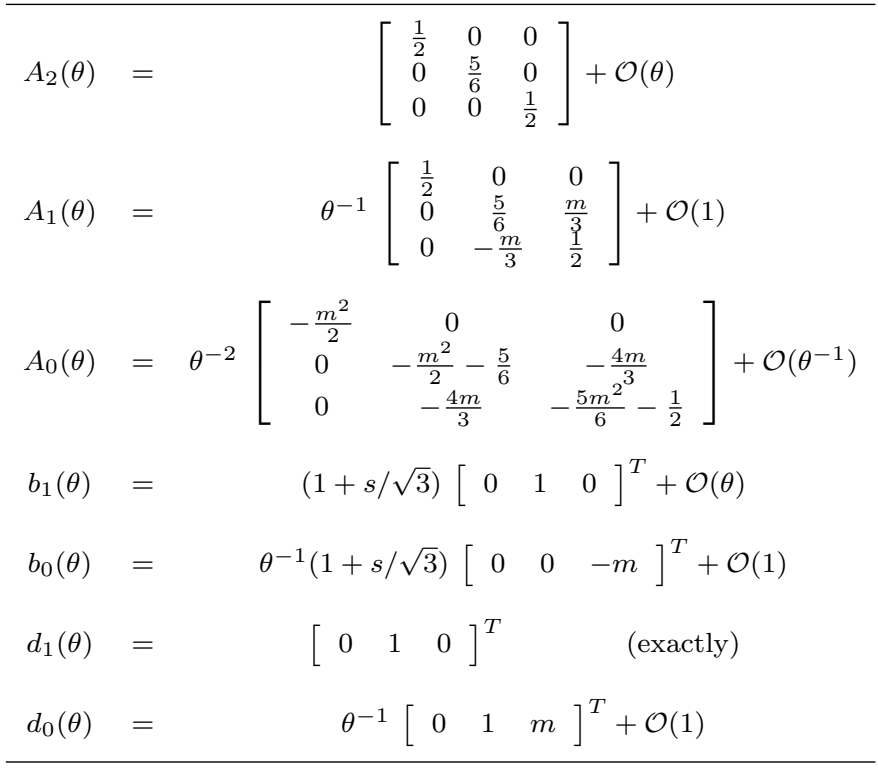

3.2. Boundary conditions at the centerline. Equations (3.17), (3.18) have a regular singular point at $\theta=0$. The leading orders of the coefficient matrices in these equations are given in Table 3.4. This information may be determined without knowing the Jenike solution explicitly since, using the fact that $\psi(0)=0$, we deduce from (3.19) that

$$
\frac{\hat{V}^{(0)}}{\hat{p}^{(0)} \hat{\lambda}^{(0)}}(0)=\frac{s}{\sqrt{3}}\left[\begin{array}{llllll}
-2 & 0 & 1 & 0 & 0 & 1
\end{array}\right]^{T}
$$

According to the method of Frobenius [3], equations (3.17), (3.18) admit solutions of the form

$$
\hat{v}^{(1)}(\theta)=\theta^{\nu} F(\theta), \quad \hat{p}^{(1)}(\theta)=\theta^{\nu-1} f(\theta),
$$

where $F(\theta)$ and $f(\theta)$ are analytic near $\theta=0$. Suppose the exponent $\nu$ is real; if $1 \leq \nu$, such a solution is continuous; if $\nu<0$, it is singular; and if $0 \leq \nu<1$, it is continuous, provided $f(0)=0$.

Proposition 3.2. There are exactly three linearly independent solutions of (3.17), (3.18) of the form (3.21) that are continuous at $\theta=0$.

Proof. Substitution of (3.21) into (3.17), (3.18) gives an indicial equation with roots

$$
\nu= \pm(m+1), \pm m, \pm(m-1)
$$

If $m \geq 2$, three of the roots of (3.22) are negative and three are positive. The three solutions associated with the positive indices are linearly independent and continuous. (We remark that since the positive roots differ by integers, in principle these solutions 
might contain log terms. This would not affect their continuity. Moreover, using Maple we have verified that no such log terms arise. See section 5.2 for more details about the Maple code.)

If $m=1$, there are four nonnegative roots of (3.22), including zero which is a double root. Because of a $\log$ term, the double root zero contributes at most one continuous solution. Again using Maple we have eliminated the various alternative possibilities to show that zero and the two positive roots (3.22) contribute exactly three linearly independent continuous solutions and these do not contain any log terms.

Incidentally, since the roots of the indicial equation are integers and since no log terms arise, the continuous solutions of the lemma are actually analytic near $\theta=0$.

Note that there are six roots (3.22) of the indicial equation. Therefore (3.17), (3.18) has a six-dimensional solution space. (We also prove this by a different argument in Corollary 4.2.) Thus, according to the proposition, the condition that solutions be regular at $\theta=0$ is equivalent to three boundary conditions. Therefore, regularity at $\theta=0$ plus the three boundary conditions (2.5), (2.6) will provide a complete set of boundary conditions.

3.3. Boundary conditions at the hopper wall. We derive the perturbed version of (2.5) in some detail; similar issues arise for (2.6), and we treat the latter equation more succinctly. The calculations are greatly simplified by the fact that we may neglect any quantity that is $\mathcal{O}\left(\epsilon^{2}\right)$. To exploit this simplification efficiently, we temporarily use the notation $F \sim G$ to mean that $F=G+\mathcal{O}\left(\epsilon^{2}\right)$.

Including a prefactor of $r^{2}$ to remove all $r$-dependence from the equation, we may rewrite $(2.5)$ as

$$
r^{2} v\left(r, \theta_{w}+\epsilon \cos m \phi, \phi\right) \cdot N=0 .
$$

Because of the perturbation, (3.23) differs from (2.10) in three respects:

- the velocity $v$ contains an additional term, $v \sim v^{(0)}+\epsilon v^{(1)}$;

- the velocity is evaluated at a location shifted by $\epsilon \cos m \phi$;

- the direction of the normal $N$ is changed.

Regarding the first two points, we observe that

$$
r^{2} v\left(r, \theta_{w}+\epsilon \cos m \phi, \phi\right) \sim \hat{v}^{(0)}\left(\theta_{w}\right)+\epsilon \cos m \phi \partial_{\theta} \hat{v}^{(0)}\left(\theta_{w}\right)+\epsilon \operatorname{trig} m \phi \hat{v}^{(1)}\left(\theta_{w}\right),
$$

where trig $m \phi$ equals $\cos m \phi$ or $\sin m \phi$, depending on the component of $\hat{v}^{(1)}$. Regarding the third point, $\partial \Omega$ is the zero set of the function $\theta-\theta_{w}-\epsilon \cos m \phi$. Taking the gradient of this function, we conclude that the (inward) normal is

$$
N \sim\left[\begin{array}{lll}
0 & -1 & -\epsilon \frac{\sin m \phi}{\sin \theta_{w}}
\end{array}\right]^{T} .
$$

Modulo an $\mathcal{O}\left(\epsilon^{2}\right)$-error, $N$ has unit length. Substituting the previous two equations into (3.23), we deduce that

$$
\begin{gathered}
-r^{2} v\left(r, \theta_{w}+\epsilon \cos m \phi, \phi\right) \cdot N \sim \hat{v}_{\theta}^{(0)}\left(\theta_{w}\right)+\epsilon \cos m \phi\left(\partial_{\theta} \hat{v}_{\theta}^{(0)}\left(\theta_{w}\right)+\hat{v}_{\theta}^{(1)}\left(\theta_{w}\right)\right) \\
+\epsilon \frac{\sin m \phi}{\sin \theta_{w}} \hat{v}_{\phi}^{(0)}\left(\theta_{w}\right) .
\end{gathered}
$$

However, since $v_{\theta}^{(0)}$ and $v_{\phi}^{(0)}$ vanish identically for Jenike's solution, the velocity boundary condition for the perturbed problem reduces to

$$
\hat{v}_{\theta}^{(1)}\left(\theta_{w}\right)=0 \text {. }
$$


We turn to the stress boundary condition (2.6). As regards the scalar $\tau_{N}$ in (2.6), we observe that, since $T_{r \phi}^{(0)}$ and $T_{\theta \phi}^{(0)}$ vanish for Jenike's solution,

$$
\tau_{N}=\sum_{i, j=1}^{3} T_{i j} N_{i} N_{j} \sim T_{\theta \theta}^{(0)}+\epsilon T_{\theta \theta}^{(1)} .
$$

The vectors $\tau_{T}$ and $v_{T}$ in (2.6) lie in a two-dimensional subspace tangent to $\partial \Omega$. Note that the unperturbed tangent space is spanned by the $r$ and $\phi$ coordinate directions. Even allowing for the perturbation, the two sides of (2.6) will be equal iff their $r$ - and $\phi$-components are equal; in symbols, iff

$$
\left[\begin{array}{c}
\tau_{T r} \\
\tau_{T \phi}
\end{array}\right]=-\frac{\mu_{w} \tau_{N}}{|v|}\left[\begin{array}{c}
v_{r} \\
v_{\phi}
\end{array}\right]
$$

This equality will hold iff (i) the two sides of the equation are parallel vectors and (ii) the first components of the two sides are equal; again, in symbols, iff

$$
\begin{aligned}
\tau_{T r} v_{\phi}-\tau_{T \phi} v_{r} & =0 \quad \text { and } \\
\tau_{T r}+\mu_{w} \tau_{N}\left(v_{r} /|v|\right) & =0 .
\end{aligned}
$$

Regarding $v$, it is clear that

$$
\left[\begin{array}{l}
v_{r} \\
v_{\phi}
\end{array}\right] \sim\left[\begin{array}{c}
v_{r}^{(0)} \\
0
\end{array}\right]+\epsilon\left[\begin{array}{c}
v_{r}^{(1)} \\
v_{\phi}^{(1)}
\end{array}\right] .
$$

Regarding $\tau_{T}=\tau-\tau_{N} N$, we claim that

$$
\left[\begin{array}{c}
\tau_{T r} \\
\tau_{T \phi}
\end{array}\right] \sim-\left[\begin{array}{c}
T_{r \theta}^{(0)} \\
0
\end{array}\right]-\epsilon\left[\begin{array}{c}
T_{r \theta}^{(1)} \\
T_{\theta \phi}^{(1)}
\end{array}\right] .
$$

Verifying this claim is straightforward except that, in analyzing the second component, one must invoke the fact that Jenike's solution satisfies $T_{\theta \theta}^{(0)}=T_{\phi \phi}^{(0)}$. On substituting (3.28) and (3.29) into (3.26), we obtain the equation

$$
\epsilon\left(T_{r \theta}^{(0)} v_{\phi}^{(1)}-v_{r}^{(0)} T_{\theta \phi}^{(1)}\right)=0 \quad \text { at } \theta=\theta_{w}+\epsilon \cos m \phi .
$$

The difference between evaluating this expression at $\theta=\theta_{w}$ and at the perturbed location is $\mathcal{O}\left(\epsilon^{2}\right)$. Removing the $r$-dependence (proportional to $r$ ) and the $\phi$-dependence (proportional to $\sin m \phi$ ) from this equation, we obtain the first stress boundary condition for the perturbed problem:

$$
\left(\hat{T}_{r \theta}^{(0)} \hat{v}_{\phi}^{(1)}-\hat{v}_{r}^{(0)} \hat{T}_{\theta \phi}^{(1)}\right)=0 \quad \text { at } \theta=\theta_{w} .
$$

Regarding (3.27), we claim that

$$
|v|=\sqrt{v_{r}^{2}+v_{\theta}^{2}+v_{\phi}^{2}} \sim\left|v_{r}\right| .
$$

Indeed, it is clear from (3.28) that the contribution of $v_{\phi}$ to $|v|$ is $\mathcal{O}\left(\epsilon^{2}\right)$, and by (3.24) the contribution of $v_{\theta}$ to $|v|$ is $\mathcal{O}\left(\epsilon^{4}\right)$. Thus, $v_{r} /|v| \sim-1$. Substituting (3.25) and (3.29) into (3.27), we obtain the condition

$$
\left(T_{r \theta}^{(0)}+\epsilon T_{r \theta}^{(1)}\right)+\mu_{w}\left(T_{\theta \theta}^{(0)}+\epsilon T_{\theta \theta}^{(1)}\right) \sim 0 \quad \text { at } \theta=\theta_{w}+\epsilon \cos m \phi .
$$


By (2.12), $T_{r \theta}^{(0)}+\mu_{w} T_{\theta \theta}^{(0)}$ vanishes at $\theta=\theta_{w}$, but at the perturbed location these terms make an $\mathcal{O}(\epsilon)$-contribution. Allowing for this contribution and eliminating the $r$ - and $\phi$-dependence, we derive the second stress boundary condition for the perturbed problem:

$$
\hat{T}_{r \theta}^{(1)}+\mu_{w} \hat{T}_{\theta \theta}^{(1)}=-\partial_{\theta}\left(\hat{T}_{r \theta}^{(0)}+\mu_{w} \hat{T}_{\theta \theta}^{(0)}\right) \quad \text { at } \theta=\theta_{w} .
$$

We have put the inhomogeneous term, which does not involve the perturbation $T^{(1)}$, on the right side of the equation. (By contrast, (3.30) and (3.24) are homogeneous.)

It is noteworthy that the perturbed boundary conditions (3.24), (3.30), (3.31) resemble (2.10), (2.11), (2.12) rather closely.

4. Numerical approximation of the two-point boundary-value problem. The coefficients in (3.17), (3.18) depend on the zeroth-order solution discussed in section 2.2. This solution can be found numerically without difficulty; see, e.g., [6], where a shooting method is used, or [10]. We will consider the zeroth-order solution as given, and we will focus on the corrections $\hat{v}^{(1)}$ and $\hat{p}^{(1)}$.

To simplify the notation before discretization, we set

$$
w=\hat{v}^{(1)}, \quad z=\frac{d}{d \theta} \hat{v}^{(1)}, \quad \text { and } \quad q=p^{(1)}
$$

and rewrite equations (3.17), (3.18) as a first-order system

$$
\left[\begin{array}{ccc}
\mathrm{I} & 0 & 0 \\
0 & -A_{2} & b_{1} \\
0 & 0 & 0
\end{array}\right]\left[\begin{array}{l}
w^{\prime} \\
z^{\prime} \\
q^{\prime}
\end{array}\right]+\left[\begin{array}{ccc}
0 & -\mathrm{I} & 0 \\
-A_{0} & -A_{1} & b_{0} \\
d_{0}^{T} & d_{1}^{T} & 0
\end{array}\right]\left[\begin{array}{l}
w \\
z \\
q
\end{array}\right]=\left[\begin{array}{l}
0 \\
0 \\
0
\end{array}\right]
$$

where the coefficient matrices are the same as above. System (4.1) is completed by the three boundary conditions (3.24), (3.30), (3.31).

The above system (4.1) is differential-algebraic; in the next lemma, we show it has index one. (The meaning of this term is defined in the proof, or see [4].) The approximation of solutions of the initial-value problem for such low-index DAEs is relatively well understood; see, for instance, [4] for convergence results. Moreover, some results for the initial-value problem may be extended to boundary-value problems; see [5]. These considerations provide a theoretical justification for our using the midpoint rule to solve (4.1) numerically.

Lemma 4.1. Assuming downward flow, i.e., $v_{r}(\theta)<0$ for any $\theta$, the first-order system is differential-algebraic of index one.

Proof. We need to show that by differentiating some of the components of (4.1) at most once, the algebraic character of the system can be eliminated, leaving a purely differential equation. Let us differentiate only the last component of (4.1),

$$
d_{0}^{T} w+d_{1}^{T} z=0
$$

The resulting system may be written as

$$
\left[\begin{array}{ccc}
I & 0 & 0 \\
0 & -A_{2} & b_{1} \\
0 & d_{1}^{T} & 0
\end{array}\right]\left[\begin{array}{c}
w^{\prime} \\
z^{\prime} \\
q^{\prime}
\end{array}\right]+\left[\begin{array}{c}
\text { linear } \\
\text { zeroth-order } \\
\text { terms }
\end{array}\right]=0
$$

We claim the coefficient matrix in (4.3) is nonsingular. Then, multiplying (4.3) by the inverse of this matrix, we obtain a purely differential equation. 
To prove the claim, it suffices to show that

$$
B=\left[\begin{array}{cc}
+\hat{\lambda}^{(0)} A_{2} & b_{1} \\
d_{1}^{T} & 0
\end{array}\right]
$$

is nonsingular, where, without changing invertibility, we have inserted a factor of $-\hat{\lambda}^{(0)}$ in the upper left, which simplifies the calculation. Let us introduce the notation $W$ for the column vector on the right-hand side of (3.19), so that $\hat{V}^{(0)} /\left(\hat{p}^{(0)} \hat{\lambda}^{(0)}\right)=s W$. Then from the definitions following (3.17), (3.18), we have $b_{1}=g_{1}+s D_{1} W$. Similarly, regarding $A_{2}$, since $\mathcal{M} G_{1}=D_{1}^{T}$, we have $\hat{\lambda}^{(0)} A_{2}=D_{1} G_{1}-\frac{1}{2}\left(D_{1} W\right)\left(D_{1} W\right)^{T}$. But

$$
D_{1} W=\left[\begin{array}{lll}
-\sin 2 \psi & \frac{1}{\sqrt{3}} \cos 2 \psi & 0
\end{array}\right]^{T}
$$

Hence matrix (4.4) equals

$$
B=\left[\begin{array}{cccc}
\frac{1}{2}-\frac{1}{2} \sin ^{2} 2 \psi & * & * & -s \sin 2 \psi \\
\frac{1}{2 \sqrt{3}} \cos 2 \psi \sin 2 \psi & * & * & 1+\frac{s}{\sqrt{3}} \cos 2 \psi \\
0 & 0 & \frac{1}{2} & 0 \\
0 & 1 & 0 & 0
\end{array}\right]
$$

where $*$ indicates elements that do not affect the invertibility of $B$. It is readily calculated that

$$
\operatorname{det} B=-\frac{1}{4}\left(\cos ^{2} 2 \psi+\frac{s}{\sqrt{3}} \cos 2 \psi\right) .
$$

As shown on p. 43 of [12], the assumption that $v_{r}^{(0)}<0$ implies that $|\psi(\theta)|<\pi / 4$, and the claim follows.

Corollary 4.2. The solution space of (4.1) has dimension six.

Proof. The solution space of (4.3), which is seven-dimensional, may be parameterized by initial values $\left[\begin{array}{lll}w\left(\theta_{0}\right) & z\left(\theta_{0}\right) & q\left(\theta_{0}\right)\end{array}\right]^{T}$. Since (4.3) was obtained from (4.1) by differentiating (4.2), we conclude that for a solution of (4.3),

$$
d_{0}^{T} w(\theta)+d_{1}^{T} z(\theta) \equiv 0 \quad \text { iff } \quad d_{0}^{T} w\left(\theta_{0}\right)+d_{1}^{T} z\left(\theta_{0}\right)=0 .
$$

Thus the solution space of (4.1) may be identified with the set of solutions of (4.3) whose initial conditions satisfy the scalar equation (4.2).

The boundary-value problem (4.1), (3.24), (3.30), (3.31) is discretized using a symmetric implicit Runge-Kutta method [2], [4]. Since the solutions are expected to behave smoothly with respect to $\theta$, the simplest of those methods, namely the midpoint rule, is chosen. In spite of being only second-order accurate, this choice is shown to be adequate below. The interval $\left(0, \theta_{w}\right)$ is divided into $N$ subintervals of size $\Delta \theta=\theta_{w} / N$, defining a uniform mesh with nodes $\theta_{i}=i \Delta \theta, i=0,1, \ldots, N$. At each grid point $\theta_{i}$ there are seven unknowns,

$$
U^{i}=\left[\begin{array}{lllllll}
w_{1}^{i} & w_{2}^{i} & w_{3}^{i} & z_{1}^{i} & z_{2}^{i} & z_{3}^{i} & q^{i}
\end{array}\right]^{T} .
$$

Since there are $N+1$ grid points, there are $7(N+1)$ unknowns in total. The midpoint 
TABLE 4.1

Numerical boundary conditions at $\theta=0$.

\begin{tabular}{|c|cccc|}
\hline$m=1$ & $w_{1}^{0}=0$ & $w_{2}^{0}+w_{3}^{0}=0$ & $z_{3}^{0}=0$ & $q^{0}=0$ \\
\hline$m \geq 2$ & $w_{1}^{0}=0$ & $w_{2}^{0}=0$ & $w_{3}^{0}=0$ & $q^{0}=0$ \\
\hline
\end{tabular}

rule for the ODE (4.1) is applied on each interval $\left[\theta_{i-1}, \theta_{i}\right], i=1, \ldots, N$, leading to $7 N$ equations for the $7(N+1)$ unknowns.

Seven additional equations are needed to close the system, and these are provided by the boundary conditions. At $\theta=\theta_{w}$, the three conditions (3.24), (3.30), (3.31) are imposed, and at $\theta=0$, the four numerical boundary conditions listed in Table 4.1 are imposed. The latter boundary conditions may be justified as follows. According to (3.21), (3.22), as $\theta \rightarrow 0$,

$$
w \sim \theta^{\nu}, \quad q \sim \theta^{\nu-1},
$$

where $\nu \geq m-1$. Thus $w(0)=0$ if $m \geq 2$, and $q(0)=0$ if $m \geq 3$. In fact, if $m=2$, direct calculation of the Frobenius solution (3.21) shows that $q(0)=0$ remains true in this case, too. If $m=1$, we refer to Lemma 3.1: by parity, $w_{1}, q$, and $z_{3}=d w_{3} / d \theta$ all vanish at $\theta=0$. The fourth boundary condition in Table 4.1 follows from the last equation in (4.1) in the limit $\theta \rightarrow 0$.

The resulting $7(N+1) \times 7(N+1)$ system has the following structure:

$$
\left[\begin{array}{ccccc}
S_{1} & R_{1} & & & \\
& S_{2} & R_{2} & & \\
& & \ddots & \ddots & \\
& & & S_{N} & R_{N} \\
B_{0} & & & & B_{w}
\end{array}\right]\left[\begin{array}{l}
U^{0} \\
U^{1} \\
\vdots \\
U^{N-1} \\
U^{N}
\end{array}\right]=\left[\begin{array}{c}
0 \\
0 \\
\vdots \\
0 \\
Q
\end{array}\right] .
$$

The last row of the above system corresponds to the implementation of the boundary conditions; the $7 \times 7$ matrices $B_{0}$ and $B_{w}$ contain the coefficients entering in the formula from Table 4.1 and (3.24), (3.30), (3.31), respectively, while $Q$ corresponds to the nonhomogeneous part of the boundary condition (3.31).

\section{Numerical results.}

5.1. Secondary circulation. We claim that, for solutions of (4.1), secondary circulation-i.e., flow tangential to the spherical cap $\{r=$ const $\}$ - may be described in terms of the stream function

$$
\Psi=\frac{1}{m r} \sin \theta \sin m \phi w_{2}(\theta) .
$$

In other words, we must show that

$$
\begin{aligned}
& v_{\theta}^{(1)}=\frac{1}{r \sin \theta} \partial_{\phi} \Psi, \\
& v_{\phi}^{(1)}=-\frac{1}{r} \partial_{\theta} \Psi .
\end{aligned}
$$

Since $v_{\theta}^{(1)}=r^{-2} w_{2}(\theta) \cos m \phi$, equation (5.1a) follows by direct differentiation. On 


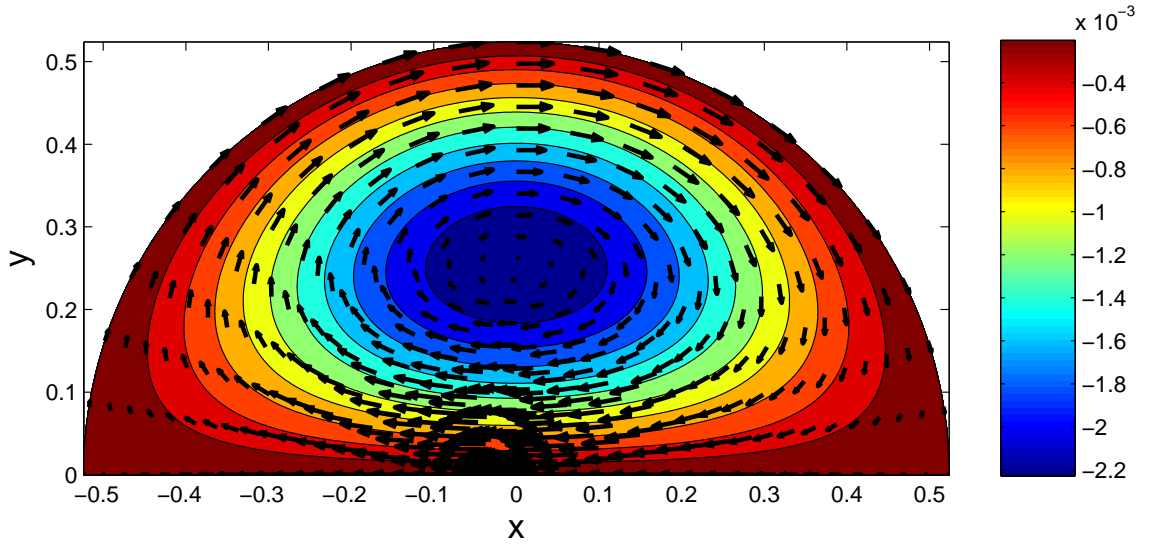

FIG. 5.1. Stream function showing secondary flow in a tilted hopper ( $m=1, \theta_{w}=30^{\circ}$, $\delta=30^{\circ}$, angle of wall friction $\left.=14^{\circ}\right)$, i.e., $\mu_{w}=\tan 14^{\circ}$. By symmetry, only half of the hopper is represented.

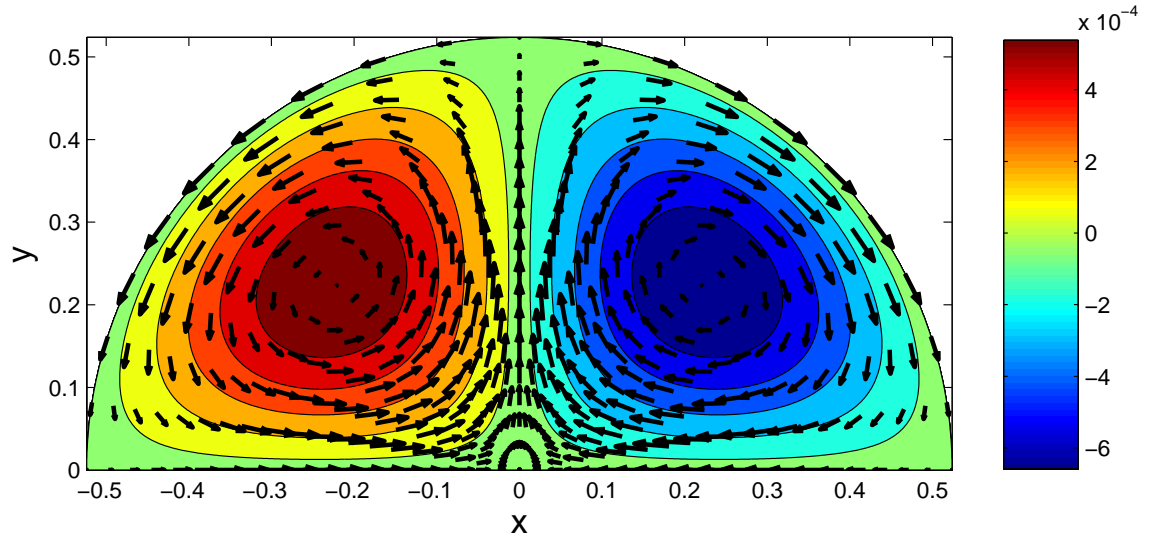

Fig. 5.2. Stream function showing secondary flow in an "elliptical" hopper $\left(m=2, \theta_{w}=30^{\circ}\right.$, $\delta=30^{\circ}$, angle of wall friction $=14^{\circ}$ ).

the other hand, since $v_{r}^{(1)}=r^{-2} w_{1}(\theta) \cos m \phi$, we have $\left(\partial_{r}+2 r^{-1}\right) v_{r}=0$, so by $(3.7)$

$$
\left(\partial_{\theta}+\cot \theta\right) w_{2}(\theta) \cos m \phi+\csc \theta w_{3}(\theta) \partial_{\phi}(\sin m \phi)=0,
$$

from which (5.1b) follows. Figures 5.1 and 5.2 show plots of the level lines of $\Psi$, which equal the projection of the streamlines onto a spherical cap $\{r=$ const $\}$. Figure 5.1 corresponds to a tilted hopper $(m=1)$, while Figure 5.2 corresponds to an "elliptical" hopper $(m=2)$. The grains do not move along radial lines but follow more complicated and fully three-dimensional trajectories.

The sign of the main circulation changes when $\mu_{w}$ increases. The corresponding transition is independent of the value of $m$, but is a property of the radial solution itself. Specifically, the circulation vanishes when the boundary condition for the correction terms (3.31) is homogeneous, i.e., $\partial_{\theta} \hat{T}_{r \theta}^{(0)}+\mu_{w} \partial_{\theta} \hat{T}_{\theta \theta}^{(0)}=0$ at $\theta=\theta_{w}$. The range of $\theta_{w}$ in Figure 5.3 is limited by the mass-flow limit - exceeding this limit leads to flows with rigid regions, to which the present model does not apply. The range of $\mu_{w}$ 


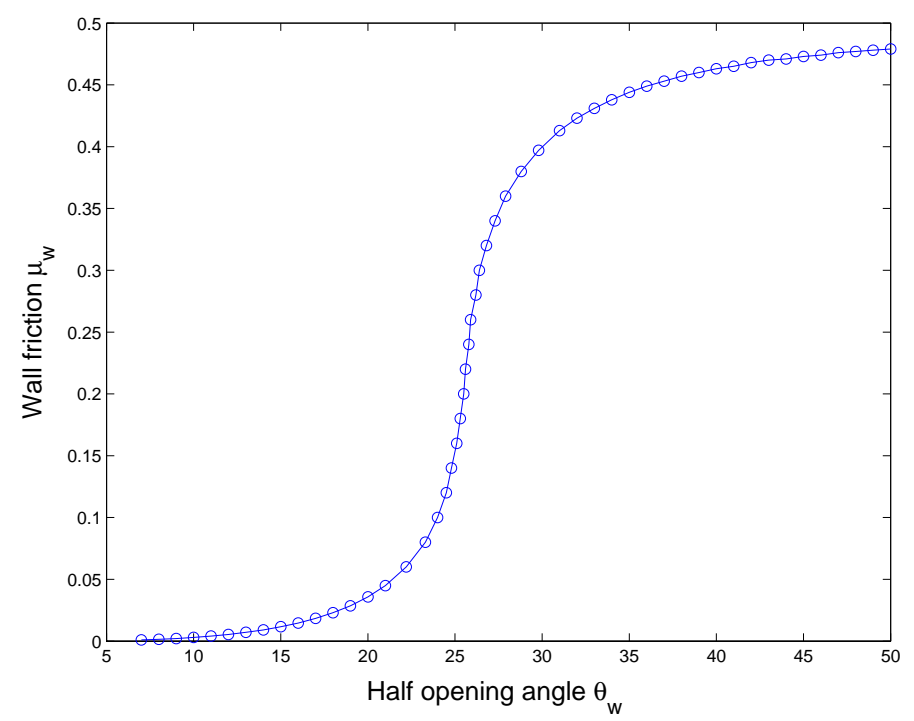

FIG. 5.3. Critical values leading to sign changes of the circulation (internal friction $\delta=30^{\circ}$ ).
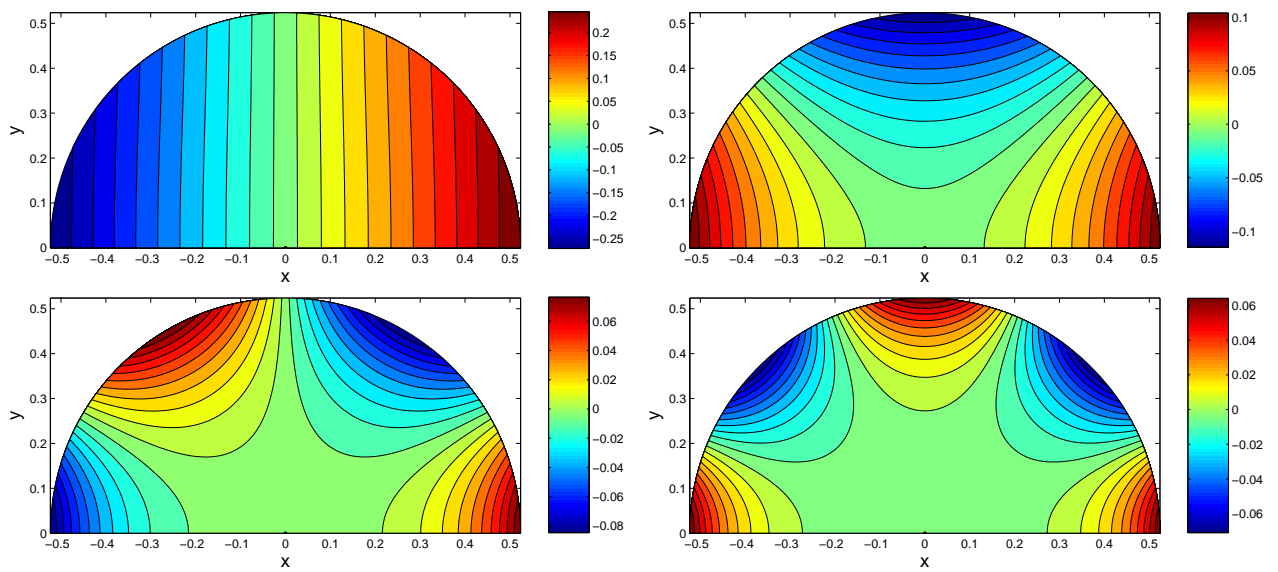

FIG. 5.4. Influence of the geometry on the mean stress corrections $\left(\theta_{w}=30^{\circ}, \delta=30^{\circ}\right.$, angle of wall friction $=14^{\circ}$ ); upper left: $m=1$, upper right: $m=2$, lower left: $m=3$, lower right: $m=4$.

is limited by the condition that $\mu_{w}<\sin \delta=1 / 2$; here the upper bound corresponds to a fully rough wall [7].

The effects of the geometry on the mean stress corrections are illustrated in Figure 5.4.

5.2. Checks on the computation. For comparison with the above numerical solution, the method of Frobenius was applied directly to the system (3.17), (3.18) using Maple. Given Jenike's radial field, a linear system for the coefficients of the series solution is readily formed and solved, yielding a solution with three free parameters, corresponding to the three linearly independent solutions in Proposition 3.2. Subsequently, the three boundary conditions (3.24), (3.30), (3.31) provide the needed relations to determine the solution to the full boundary-value problem. 

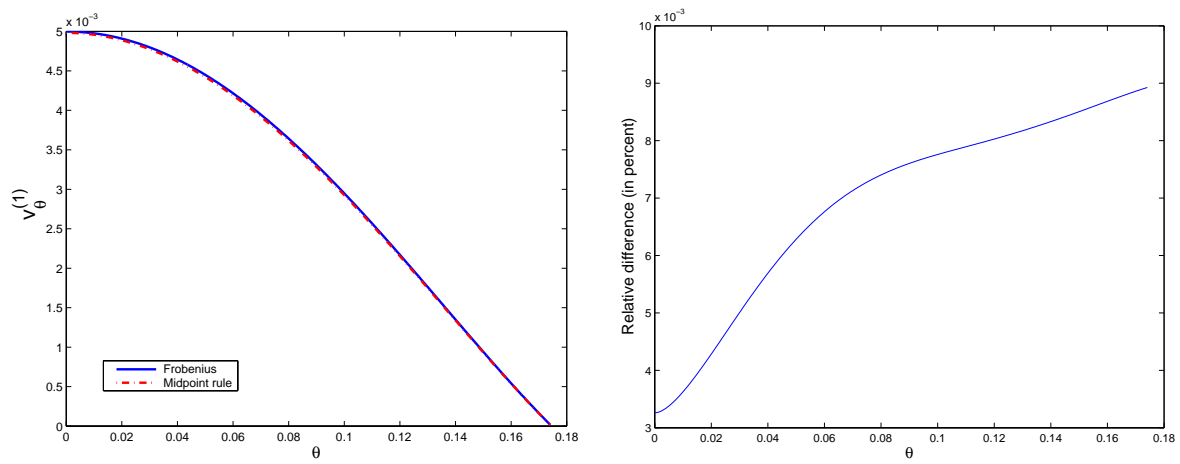

FIG. 5.5. Comparison of $v_{\theta}^{(1)}$ from the purely numerical method of section 4 and from the Frobenius method of section 5.2. (Using $m=1, \theta_{w}=10^{\circ}, \delta=30^{\circ}$, and $\mu_{w}=0.3$.)

Two methods of obtaining the radial field were employed. Under the assumption that $\theta_{w}^{2}$ and $\mu_{w} / \theta_{w}$ are both small and of the same order, a series representation of the Jenike field was computed within Maple itself. Under the less restrictive assumption that only $\theta_{w}$ be small (say $10^{\circ}$ ), numerical solutions were computed in MATLAB, fitted to polynomials, and then imported into Maple. In both cases, the resulting polynomials were then used to compute the first-order correction. The corrections to the stress and velocity obtained through this symbolic approach agree extremely well with the results of the purely numerical method of sections 4 and 5: for the representative values $m=1, \theta_{w}=10^{\circ}, \delta=30^{\circ}$, and $\mu_{w}=0.3$, the corrections obtained by the two different methods have a relative difference of less than $1 \%$; see Figure 5.5.

Acknowledgments. The authors thank Bob Behringer, Steve Campbell, Tim Kelley, Tony Royal, and Michael Shearer for many helpful discussions.

\section{REFERENCES}

[1] S. Agmon, A. Douglis, And L. Nirenberg, Estimates near the boundary for solutions of elliptic partial differential equations satisfying general boundary conditions II, Comm. Pure Appl. Math., 17 (1964), pp. 35-92.

[2] U.M. Ascher And L.R. Petzold, Computer Methods for Ordinary Differential Equations and Differential-Algebraic Equations, SIAM, Philadelphia, 1998.

[3] C.M. Bender and S.A. Orszag, Advanced Mathematical Methods for Scientists and Engineers, International Series in Pure and Applied Mathematics, McGraw-Hill, New York, 1978.

[4] K.E. Brenan, S.L. Campbell, and L.R. Petzold, Numerical Solution of Initial-Value Problems in Differential-Algebraic Equations, Classics Appl. Math. 14, SIAM, Philadelphia, 1996.

[5] K.D. Clark and L.R. Petzold, Numerical solution of boundary value problems in differentialalgebraic systems, SIAM J. Sci. Statist. Comput., 10 (1989), pp. 915-936.

[6] P.A. Gremaud, J.V. Matthews, and M. Shearer, Similarity solutions for granular materials in hoppers, in Nonlinear PDE's, Dynamics, and Continuum Physics, Contemp. Math., 255, J. Bona, K. Saxton and R. Saxton, eds., AMS, Providence, RI, 2000, pp. 79-95.

[7] A.W. Jenike, Gravity flow of bulk solids, Bulletin 108, Utah Eng. Expt. Station, University of Utah, Salt Lake City, 1961.

[8] T.M. Knowlton, J.W. Carson, G.E. Klinzing, and W.C. Yang, The importance of storage, transfer and collection, Chem. Eng. Prog., 90 (1994), pp. 44-54.

[9] E.W. Merrow, A quantitative assessment of REDD requirements for solids processing technology, Publication R-3216-DOE/PSSP, Rand Corporation, Santa Monica, CA, 1986. 
[10] R.M. Nedderman, Static and Kinematic of Granular Materials, Cambridge University Press, Cambridge, UK, 1992.

[11] N. Rege, Computational Modeling of Granular Materials, Ph.D. thesis, Department of Civil and Environmental Engineering, Massachusetts Institute of Technology, Cambridge, MA, 1996.

[12] D.G. SCHAEFFER, Instability in the evolution equations describing incompressible granular flow, J. Differential Equations, 66 (1987), pp. 19-50.

[13] J.R. Williams AND N. REgE, The development of circulation cell structures in granular materials undergoing compression, Powder Technol., 90 (1997), pp. 187-194. 
Copyright of SIAM Journal on Applied Mathematics is the property of Society for Industrial and Applied Mathematics and its content may not be copied or emailed to multiple sites or posted to a listserv without the copyright holder's express written permission. However, users may print, download, or email articles for individual use. 
Copyright of SIAM Journal on Applied Mathematics is the property of Society for Industrial and Applied Mathematics and its content may not be copied or emailed to multiple sites or posted to a listserv without the copyright holder's express written permission. However, users may print, download, or email articles for individual use. 